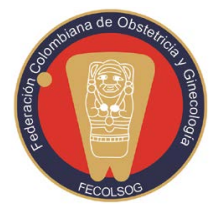

Revista Colombiana de Obstetricia y Ginecología Vol. 67 No. 4 • Octubre-Diciembre 2016 • (299-304)

\title{
ESTUDIO DE CONCORDANCIA ENTRE DOS PRUEBAS: ALFA MICROGLOBULINA PLACENTARIA TIPO 1 (PAMG-1) FRENTE A FACTOR DE CRECIMIENTO SIMILAR A LA INSULINA TIPO 1 (IGFBP-1), EN PACIENTES CON DIAGNÓSTICO INCIERTO DE RUPTURA DE MEMBRANAS, MEDELLÍN, COLOMBIA
} Concordance study between placental allpha microglobullin-1 (PAMG-1) and insullin-like growth factor-1 (IGFBP-1) tests in patients with uncertain diagnosis of rupture of membranes, Medellín, Colombia

\section{Hernán Cortés-Yepes, $M D^{I}$}

Recibido: marzo 9/16 - Aceptado: noviembre 21/16

\section{RESUMEN}

Objetivo: evaluar la concordancia diagnóstica entre dos pruebas: alfa microglobulina placentaria tipo 1 (PAMG-1) frente a factor de crecimiento similar a la insulina tipo 1 (IGFBP-1), en pacientes con diagnóstico incierto de ruptura de membranas.

Materiales y métodos: estudio de concordancia diagnóstica, de corte transversal, en mujeres embarazadas que consultaron con sospecha clínica de ruptura de membranas al Hospital Universitario San Vicente Fundación de Medellín (HUSVF), entre agosto del 2014 a diciembre de 2015, en quienes se compararon dos pruebas diagnósticas para determinar ruptura de membranas. Se excluyeron las pacientes en trabajo de parto avanzado. Se estimó

1 Ginecólogo y Obstetra; especialista en Medicina Fetal. Docente, Universidad de Antioquia, Hospital Universitario de San Vicente Fundación, Medellín (Colombia). hcortes74@hotmail.com la concordancia en el resultado de las pruebas respecto a la presencia o no de ruptura de membranas a través del índice de Kappa.

Resultados: de 83 pacientes, 27,7\% dieron positivas para ruptura prematura de membranas (RPM) con la prueba PAMG-1 (Amnisure) y 28,9\% con la IGFBP-1 (Actim-Prom); el único caso dispar resultó positivo a la amnionfusión con índigo de carmín. La concordancia entre ambas pruebas fue de 0,97. Conclusión: la IGFBP-1 demostró un desempeño similar a la PAMG-1 para el diagnóstico de ruptura de membranas.

Palabras clave: ruptura prematura de membranas fetales, diagnóstico, pruebas diagnósticas, carmín de índigo.

\section{ABSTRACT}

Objective: To assess diagnostic concordance between placental alpha macroglobulin-1 (PAMG- 
1) and insulin-like growth factor-binding protein 1 (IGFBP-1) tests in patients with uncertain diagnosis of rupture of membranes.

Materials and methods: Diagnostic concordance cross-sectional study in pregnant women with clinical suspicion of rupture of membranes seen at Hospital Universitario San Vicente Fundación de Medellín (HUSVF) between August 2014 and December 2015, in whom two diagnostic tests for the diagnosis of rupture of membranes were compared. Patients in advanced labour were excluded. Concordance of test results was estimated in terms of the presence or not of ruptured membranes, using the Kappa Index.

Results: Of 83 patients, $27.7 \%$ were positive for rupture of membranes when the PAMG-1 (Amnisure) was used and 28.9\% were positive with the IGFBP-1 (Actim-Prom) test; the only case of disparity was positive to amniotic infusion of indigo carmine. Concordance between both tests was 0.97.

Conclusion: The performance of IGFBP-1 was similar to that of PAMG-1 for diagnosing rupture of membranes.

Key words: Premature rupture of membranes, diagnosis, diagnostic tests, indigo carmine.

\section{INTRODUCCIÓN}

La ruptura prematura de membranas (RPM) se define como la pérdida de la continuidad de las membranas ovulares antes del inicio del trabajo de parto y se presenta en el 1-5\% de los embarazos; es la responsable de al menos un tercio de los partos prematuros, y se asocia con corioamnionitis, compresión del cordón umbilical, abruptio de placenta, endometritis posparto y otras complicaciones dependiendo de la edad gestacional a la que se presente $(1,2)$.

En cerca del $90 \%$ de los casos el diagnóstico se confirma con base en la historia clínica y el examen físico; sin embargo, en el resto estos son dudosos y se debe recurrir a otras pruebas para confirmar la sospecha (3).
La amnioinfusión con índigo de carmín es considerada por algunos autores como el patrón de oro (gold standard) para el diagnóstico de la RPM; no obstante, este procedimiento es invasivo, costoso y puede provocar la ruptura de las membranas y otras complicaciones, como infecciones (4-7). Aunque en las últimas décadas, debido a los avances en las técnicas y los equipos de ultrasonido, se ha mejorado la tasa de éxito de la amnioinfusión y se ha disminuido el número de complicaciones, esta no se utiliza de manera rutinaria (8).

Debido a las limitaciones y los riesgos del patrón de oro, se han elaborado otras pruebas que detectan sustancias que solo están presentes en el líquido amniótico (factor de crecimiento similar a la insulina unido a la proteína 1 [IGFBP-1] y la alfa microglobulina placentaria tipo 1 [PAMG-1]), por lo que su detección en las secreciones vaginales es indicativa de RPM (9).

La IGFBP-1 (nombre comercial: ACTIMPROM) es una proteína de $28 \mathrm{kDa}$ producida por el hígado fetal y la decidua, que está presente en el líquido amniótico en altas concentraciones, pero ausente en el plasma, el semen, la sangre o la orina. Su concentración en el líquidio amniótico aumenta con la edad gestacional, alcanzando niveles de $145.000 \mathrm{ng} / \mathrm{ml}$ al término; el límite de detección de la prueba Actim Prom es de 25 ng/ml (9). La sensibilidad de esta prueba varía del 74 al $100 \%$ y su especificidad del 77 al $98 \%$ (10-13).

La PAMG-1 (nombre comercial Amnisure) es una glicoproteína de $34 \mathrm{kDa}$ y es producida por la decidua, su concentración en el líquido amniótico oscila de 2000 a $25.000 \mathrm{ng} / \mathrm{ml}$, mientras que en sangre materna no supera los $2 \mathrm{ng} / \mathrm{ml}$. El Amnisure tiene un límite de detección de la PAMG-1 de $5 \mathrm{ng} / \mathrm{ml}$ y la sensibilidad reportada es cercana al $99 \%$, con una especificidad que varía del 87-100\% (14-16). Algunos trabajos han demostrado que la presencia de sangre, semen y orina no alteran el resultado de esta prueba (17). 
Aunque ambas pruebas han sido comparadas contra la amnioinfusión con índigo carmín (patrón de oro) y han demostrado ser útiles en el diagnóstico de RPM $(16,18)$, solo un número limitado de estudios de calidad han evaluado cuál de las dos es la mejor y no se conoce la concordancia entre las dos pruebas en pacientes con RPM y en especial en aquellas con un diagnóstico dudoso.

Por tanto, el objetivo de este estudio fue evaluar la concordancia diagnóstica entre el PAMG-1 y la IGFBP-1 en las pacientes con diagnóstico dudoso de ruptura prematura de membranas.

\section{MATERIALES Y MÉTODOS}

Estudio de concordancia diagnóstica, de corte transversal, en mujeres embarazadas que consultaron con sospecha clínica de ruptura de membranas al Hospital Universitario San Vicente Fundación de Medellín (HUSVF) entre agosto del 2014 a diciembre de 2015; se excluyeron las pacientes en trabajo de parto activo (dilatación mayor de $4 \mathrm{~cm}$ ). El HUSVF es un hospital general de alta complejidad y centro de referencia del departamento de Antioquia, que atiende población de alto riesgo afiliada principalmente al régimen subsidiado por el Estado.

Se realizó muestreo consecutivo por conveniencia, debido a que se contaba con 90 pruebas (Actim-Prom) que fueron donadas por el fabricante, el Amnisure es la prueba que se realiza de manera rutinaria en el Hospital cuando existen dudas en el diagnóstico.

Procedimiento. Las mujeres gestantes que consultaron al servicio de urgencias del Hospital con sospecha de ruptura de membranas, pero cuyo diagnóstico no se pudo determinar por medio del examen físico y la ecografía, fueron invitadas a participar en el estudio. Se les brindó información sobre el objetivo del mismo y se les solicitó el consentimiento informado para su inclusión en el mismo. Posteriormente, se aplicó un formulario para registrar las variables sociodemográficas y clínicas; luego se tomaron dos muestras vaginales con hisopo y con ayuda de un espéculo desechable, una para cada prueba, siguiendo las indicaciones del fabricante; se realizaba primero la PAMG-1 y luego la IGFBP-1, las cuales se leían como positivas si se veían 2 líneas rojas, y negativas si solo aparecía una (la línea superior de validación); o como indeterminada, si no se veía ninguna línea o solo aparecía la línea inferior (sin la línea superior de validación). En caso de disparidad en los resultados, se realizó una amnioinfusión con índigo de carmín.

Al médico tratante solo se le informó el resultado de la prueba PAMG-1 (Amnisure), que es la prueba que se usa de manera rutinaria en el servicio (o del índigo en los casos de disparidad); esta prueba confirmaba el diagnóstico de ruptura, por lo que se iniciaba el manejo de RPM según el protocolo establecido en la institución. Las pacientes con dos pruebas negativas fueron consideradas sin ruptura de membranas. Las pacientes a quienes se les descartó la RPM fueron dadas de alta y continuaron su control prenatal habitual. Las mujeres gestantes en las que se confirmó el diagnóstico por medio de la clínica o la ecografía, se hospitalizaron y se inició el manejo establecido en los protocolos de la institución. El investigador (HCY) era el único que conocía el resultado de las dos pruebas ya que era el encargado de realizarlas.

Se midieron las siguientes variables: edad materna, edad gestacional al diagnóstico, paridad, índice de líquido amniótico (ILA), resultados de las pruebas rápidas y de la amnioinfusión en los casos en que las pruebas rápidas fueron dispares; también se indagó por el resultado perinatal de aquellos casos en que se confirmó la ruptura de membranas (edad gestacional al parto, peso y vía del parto).

Análisis. Las variables cuantitativas se presentan con media o mediana, con su respectiva medida de dispersión, desviación estándar o percentiles 25 y 75 (p25-75). Se calculó la concordancia entre ambas pruebas usando el índice kappa, con su significancia respectiva. La base de datos se elaboró en el programa Excel y los análisis estadísticos usando los programas SPSS 20 y Epidat 3.1.

Aspectos éticos. Este protocolo contó con la aprobación del comité de ética del HUSVF y el consen- 
timiento informado de las mujeres participantes; se garantizó la confidencialidad de las pacientes.

\section{RESULTADOS}

Un total 83 gestantes cumplieron con los criterios de selección. Se realizaron ambas pruebas y en todos los casos se conoció el resultado de las mismas, pero en 9 (10\%) se encontraron datos incompletos en algunas de las variables sociodemográficas de base.

La media de la edad materna fue de 23,8 años (DS $\pm 6,86$ ), siendo la menor de 13 y la mayor de 44; la mediana de la edad gestacional fue de 32,2 semanas (p25-75: 30-35), la mediana del número de hijos fue 2 (p25-75: 0-3) y la del ILA al momento de las pruebas de 10 (p25-75).

De las 83 pacientes, 23 (27,7\%) dieron positivas para ruptura de membranas (RPM) con la prueba PAMG-1 (Amnisure) y 24 (28, $9 \%$ ) con la IGFBP-1 (Actim-Prom); el único caso dispar fue sometido a amnioinfusión con índigo de carmín, con resultado positivo. En 59 gestantes ambas pruebas fueron negativas (tabla 1). El índice de concordancia kappa fue de $0,970(\mathrm{p}=0,000)$.

El ILA promedio en las pacientes con prueba rápida positiva fue de 6,2 , y de 10,4 en promedio en aquellas con prueba rápida negativa.

La edad gestacional promedio al momento de la terminación del embarazo en las pacientes a quienes se les confirmó la RPM fue de 31,2 semanas; el peso promedio de los recién nacidos fue de $1880 \mathrm{~g}$ y fallecieron los 3 que nacieron antes de las 24 semanas; se realizaron 9 cesáreas $(42,8 \%)$ y se atendieron 12 partos vaginales. Tres pacientes tenían un embarazo gemelar y en dos se confirmó la ruptura de uno de los sacos.

No se obtuvieron los datos de tres pacientes atendidas en otra institución y que solo fueron remitidas para la realización de las pruebas.

\section{DISCUSIÓN}

En este trabajo se encontró que tanto la IGFBP-1 (Actim-Prom) como la PAMG-1 (Amnisure) tienen un desempeño similar para el diagnóstico de ruptura de membranas en pacientes con diagnóstico incierto, pues se encontró una concordancia de 0,970, que se considera como muy buena (19), y aunque no se compararon contra el patrón de oro, otros trabajos lo han hecho y han concluido que ambas tienen buena sensibilidad y pueden ser una alternativa útil y no invasiva para el diagnóstico.

Es poca la información disponible sobre la concordancia de las pruebas en estudio en la literatura internacional. Liang et al. informan una concordancia de 0,93 entre la IGFBP-1 y la PAMG-1 en un total de 120 pacientes entre 11 y 42 semanas (20).

Sosa et al., al comparar la PAMG-1 (Amnisure) con índigo carmín en 140 pacientes para el diagnóstico de RPM en casos dudosos, con una prevalencia de RPM del 19\%, encontraron que dicha prueba tenía una sensibilidad del $100 \%$ y una especificidad del 99,1\%, concluyendo que es una alternativa útil y sensible en dichos casos (16). Cuartas et al. compararon en 178 mujeres la utilidad del IGFBP-1 (Actim-Prom) contra el índigo carmín en los casos

\section{Tabla 1.}

Resultados de pruebas rápidas (PAMG-1 frente a IGFBP-1) en mujeres con sospecha de ruptura prematura de membranas en el Hospital San Vicente de Paul de Medellín, Colombia, 2014-2015

IGFBP-1 (Actim-Prom)

\begin{tabular}{|l|c|c|c|c|}
\hline & & + & - & Total \\
\hline $\begin{array}{l}\text { PAMG-1 } \\
\text { (Amnisure) }\end{array}$ & + & 23 & 1 & 24 \\
\hline Total & - & 0 & 59 & 59 \\
\hline
\end{tabular}


de diagnóstico dudoso y con una prevalencia del $11 \%$ para RPM, encontrando una sensibilidad del $89,4 \%$, una especificidad del 92,8\%, concluyendo que se puede usar en la práctica clínica para el diagnóstico de RPM (18).

Varios autores han comparado ambas pruebas entre sí, mostrando una sensibilidad similar entre ambas y, en algunos casos, con mejor desempeño para la PAMG-1 pero, al igual que en este trabajo, no se han comparado contra el patrón de oro. Pollet et al. compararon ambas pruebas usando diluciones de líquido amniótico en solución salina, obtenido en cesáreas electivas, demostrando que el PAMG-1 era positivo a diluciones mayores; sin embargo, no es clara la repercusión clínica de dicho hallazgo (21); Tagore et al. también compararon dichas pruebas contra nitrazina y resultado clínico (usándolos como patrón de oro), encontrando una especificidad y sensibilidad, para PAMG-1 de 100 y 92,7\%, respectivamente; para IGFBP-1 de 94,4 y 87,5\%, y para la nitrazina de 39,7 y $85 \%$, concluyendo que el PAMG-1 (Amnisure) es el más preciso y con mejor desempeño (22), aunque la diferencia no fue estadísticamente significativa y no es claro como confirmaron los casos discordantes.

Entre las debilidades de este trabajo está el tamaño de la muestra, y que no todas fueron comparadas contra el patrón de oro (índigo carmín), solo los casos discordantes; sin embargo, se logró el objetivo de este estudio que era comparar la concordancia entre dichas pruebas.

\section{CONCLUSIÓN}

La IGFBP-1 demostró un desempeño similar a la PAMG-1 para el diagnóstico de ruptura de membranas, en las pacientes con historia clínica dudosa.

\section{AGRADECIMIENTOS}

Al doctor Oscar A. Villada, epidemiólogo de la Unidad de Investigaciones del Hospital Universitario San Vicente Fundación, por su asesoría metodológica, ayuda en el análisis estadístico y revisión de este manuscrito.

\section{REFERENCIAS}

1. ACOG. Premature rupture of membranes. ACOG Practice Bulletin No. 80. American College of Obstetricians and Gynecologists. Obstet Gynecol. 2007;109:1007-19.

2. Mercer BM. Preterm Premature Rupture of the Membranes. Obstet Gynecol. 2003;101:178-93.

3. Cortés H. Ruptura prematura de membranas. En: Cortés H, Gómez J, Gutiérrez J. Obstetricia: aspectos claves. CIB; 2013.

4. Guevara AM, Vergara MI, Gutiérrez JH, Sanín JE. Amnioinfusión con índigo carmín en sospecha de ruptura prematura de membranas ovulares pretérmino. Rev Colomb Obstet Ginecol. 2007;58:237-42.

5. Fujimoto S, Kishida T, Sagawa T, Negishi H, Okuyama $\mathrm{K}$, Hareyama H, et al. Clinical usefulness of the dyeinjection method for diagnosing premature rupture of the membranes in equivocal cases. J Obstet Gynaecol (Tokyo). 1995;21:215-20.

6. McEnerney JK, McEnerney LN. Unfavorable neonatal outcome after intraamniotic injection of methylene blue. Obstet Gynecol. 1983;61(3 Suppl):35S-37S.

7. Smith RP. A technic for the detection of rupture of the membranes. A review and preliminary report. Obstet Gynecol. 1976;48:172-6.

8. Simhan HN, Canavan TP. Preterm premature rupture of membranes: diagnosis, evaluation and management strategies. BJOG. 2005;112(Suppl 1):32-7.

9. Di Renzo GC, Roura LC, Facchinetti F, Antsaklis A, Breborowicz G, Gratacos E, et al. Guidelines for the management of spontaneous preterm labor: identification of spontaneous preterm labor, diagnosis of preterm premature rupture of membranes, and preventive tools for preterm birth. J Maternal Fetal Neonatal Med. 2011;24:659-67. doi: 10.3109/14767058.2011.553694.

10. Rutanen EM, Karkkainen TH, Lehtovirta J, Uotila J, Hinkula MK, Hartikainen AL. Evaluation of a rapid strip test for insulinlike growth factor-binding protein-1 in the diagnosis of ruptured of fetal membrane. Clin Chim Acta. 1996; 253:91-101.

11. Chen FC, Dudenhausen JW. Comparison of two rapid strip tests based on IGFBP-1 and PAMG-1 
for the detection of amniotic fluid. Am J Perinatol. 2008;25:243-6. doi: 10.1055/s-2008-1066876.

12. Erdemoglu E, Mungan T. Significance of detecting insulinlike growth factor binding protein-1 in cervicovaginal secretions: comparison with nitrazine test and amniotic fluid volume assessment. Acta Obstet Gynecol Scand. 2004;83:622-6.

13. Jeurgens-Borst AJ, Bekkers RL, Sporken JM, van der Berg PP. Use of insulin like growth factor binding protein-1 in the diagnosis of ruptured fetal membranes. Eur J Obstet Gynecol Reprod Biol. 2002;102:11-4.

14. Cousins LM, Smok DP, Lovett SM, Poeltler DM. Amnisure placental a microglobulin-1 rapid immunoassay versus standard diagnostic methods for detection of rupture of membranes. Am J Perinatol. 2005;22:317-20.

15. Lee SE, Park JS, Norwitz ER, Kim KW, Park HS, Jun JK. Measurement of placental a-microglobulin-1 in cervicovaginal discharge to diagnose rupture of membranes. Obstet Gynecol. 2007;109:634-40.

16. Sosa CG, Herrera E, Restrepo JC, Strauss A, Alonso J. Comparison of placental alpha microglobulin-1 in vaginal fluid with intra-amniotic injec-tion of indigo carmine for the diagnosis of rupture of membranes. J Perinat Med. 2014;42:611-6.

17. Wilfong L. Effects of maternal blood on the readability and reliability of the amnisure rapid immunoassay. Med Educ Foundation Am Coll Osteopathic Obstet Gynecol Winter Newslett. 2009; 30:11-2.
18. Cuartas A, Villa J, Cortés H, Tamayo J, Ossa B, Jaramillo A, et al. Diagnosis of preterm rupture of membranes with a rapid test that detects insulin-like growth factor binding protein 1 (IGFBP-1) and a comparison with indigo carmine amnioinfusion (en proceso de publicación).

19. Cortés-Reyes E, Rubio-Romero JA, Gaitán-Duarte H. Métodos estadísticos de evaluación de la concordancia y la reproducibilidad de pruebas diagnósticas. Rev Colomb Obstet Ginecol. 2010;61:247-55.

20. Liang DK, Qi HB, Luo X, Xiao XQ, Jia XY. Comparative study of placental $\alpha$-microglobulin-1, insulin-like growth factor binding protein-1 and nitrazine test to diagnose premature rupture of membranes: a randomized controlled trial. J Obstet Gynaecol Res. 2014;40:1555-60. doi: 10.1111/ jog. 12381 .

21. Pollet-Villard M, Cartier R, Gaucherand P, Doret M. Detection of Placental Alpha Microglobulin-1 versus Insulin-Like Growth Factor-Binding Protein-1 in Amniotic Fluid at Term: A Comparative Study. Am J Perinatol. 2011;28:489-94.

22. Tagore S, Kwek K. Comparative analysis of insulin-like growth factor binding protein-1 (IGFBP-1), placental alpha microglobulin-1 (PAMG-1) and nitrazine test to diagnose premature rupture of membranes in pregnancy. J Perinat Med. 2010;38:609-12. doi: 10.1515/JPM.2010.099.

Conflicto de intereses: este trabajo se realizó con pruebas donadas por el laboratorio DIMA Gesellschaft für Diagnostika mbH (DIMA GmbH), Robert-Bosch-Breite 23. 37079 Goettingen. Germany.

El autor no ha recibido ninguna remuneración por la realización de este trabajo, ni tiene ninguna relación con los fabricantes de las pruebas. 